\title{
16 例创伤性湿肺的诊治分析
}

\author{
马俊涛 魏国清 \\ 唐山中心医院 \\ DOI:10.32629/bmtr.v1i1.323
}

[摘 要] 目的：探讨创伤性湿肺诊治经验。方法：回顾性分析我院重症医学科2018年10月至2019年10月收治的16例创伤性湿肺的患者,入院后 明确诊断,给予气管插管, 机械通气, 采用小潮气量肺保护性通气策略, 积极给予原发伤处理、抗休克, 吸氧、镇痛镇静、抗炎、化痰及支气管扩张 等治疗,并在患者脱机拔管前采用限制性液体管理策略进行输液治疗。结果: 16例患者均顺利脱机拔管转出重症监护病房。结论: 创伤性湿肺 患者在采取原发伤及基础治疗的同时采用有创机械通气联合限制性液体管理策略综合治疗,效果较好。

[关键词] 创伤性湿肺; 有创机械通气; 限制性液体管理策略; 综合治疗

\section{Diagnosis and Treatment of 16 Cases of Traumatic Wet Lung \\ Ma Juntao Wei Guoqing \\ Tangshan Central Hospital}

[Abstract] 0bjective: To explore the experience of diagnosis and treatment of traumatic wet lung. Methods: Retrospective analysis of 16 patients with traumatic wet lung admitted to our Department of Critical Care Medicine from 0ctober 2018 to 0ctober 2019, after admission, the diagnosis was confirmed, endotracheal intubation, mechanical ventilation, and a small tidal volume pulmonary protective ventilation strategy, actively give primary wound treatment, anti-shock, oxygen, analgesic sedation, anti-inflammatory, phlegm and bronchiectasis treatment, and infusion therapy with restrictive fluid management strategy before the patient is directly extubated. Results: All 16 patients were successfully removed from the intensive care unit offline. Conclusion: In patients with traumatic wet lung, the combination of invasive mechanical ventilation and restrictive fluid management strategy is effective in the treatment of primary injury and basic treatment.

[Key words] traumatic wet lung; invasive mechanical ventilation; restrictive fluid management strategy; comprehensive treatment

创伤性湿肺是因患者胸部受损或全身多部位创伤, 激活患者的神经应激反应, 不单指肺部单纯损伤, 也指患者器官部位损伤后导致的神经应 激反应, 这种病理改变会激活全身炎症反应, 释放一系列毒素及炎症因子, 包括白介素、组胺、中性粒细胞因子及血小板活化产物, 中性粒细胞是重 要的炎症细胞, 它不仅可以产生大量炎症因子使肺组织损伤, 可以作为信 号分子激活炎症细胞及炎症级联反应, 造成肺内炎症反应失控, 是炎症反 应的启动因子。如 IL-1 $\beta$ 、IL-6、IL-8、TNF- $\alpha$ 等, 这些炎症因子继而导 致中性粒细胞的激活, 成恶心循环。继而产生炎症反应的 “瀑布效应” ${ }^{[1]}$ 。 上述毒素可导致患者全身血管炎症, 累计全身各个器官, 肺脏组织特点是 血管较丰富, 组织疏松, 正是由于其特点, 肺脏组织首先出现病理改变, 其 病理改变为血管炎症反应使血管扩张, 通透性增加, 使血液成分不能保留 于患者血管中, 肺脏全部毛细血管大量血浆成分外渗, 造成毛细血管渗漏
综合征。这种病理改变会导致患者肺间质水肿, 根据患者肺水肿程度, 表现 为各种程度的低氧血症级呼吸困难症状, 进一步发展会导致心力衰竭及多 器官功能衰竭, DIC, 病死率达 $40 \%-50 \%$ 。我院2018年10月至2019年10月收治 的16例重度创伤性湿肺, 治疗效果较好, 现总结如下:

\section{1 资料与方法}

1. 1 一般资料

选取我院2018年10月至2019年10月收治的16例创伤性湿肺的患者, 16 例患者中男性 9 例, 女性7例, 年龄 $40 \sim 50$ 岁, 平均年龄 45 岁。挤压伤 3 例, 交通 伤10例, 高处队落伤 3 例, 16例均有肋骨骨折; 肺挫伤并血气胸10例; 伴脑损 伤者 2 例; 合并腹部损伤者6例; 骨盆骨折者3例。伤后就诊时间: $1 \mathrm{~h} \sim 24 \mathrm{~h}$ 。 通过胸片、胸部CT三维重建检查诊断明确。

\section{2诊断依据}

\section{3 结语}

脑力劳动或体力劳动的效率和作业能力都会受到噪声的不良影响, 尤 其是从事困难而复杂的工作时, 噪声的影响就更大。因此, 企业和员工要高 度重视噪声对安全生产的不利影响, 积极主动地采取预防性措施, 把不利 降到最低程度, 确保安全生产。

[参考文献]

[1]许奸,张革建,竭雪懿.降低机械加工设备噪音方法初探[J].吉林劳 动保护,2018,(01):24-26.

[2]温武略,李伟明,谢连府.生产性噪声对作业工人心血管系统的影响 [J].中国城乡企业卫生,2019,34(03):89-90.

[3]唐伟军.减少机械噪音的方法[J].内燃机与配件,2018,(13):179-180. 
16例患者外伤病史明确, 外伤多为伤情较重, 多部位复合损伤, 患者创 伤后均出现呼吸困难, 表现为缺氧症状, 烦躁不安、部分患者咯血性泡沫痰, 不能用心力衰竭或容量过负荷解释的肺水肿征象。查体患者肺部均可闻及 大量干、湿性啰音及哮鸣音。胸部影像胸片或CT扫描, 可见双侧阴影且不 能完全用胸腔积液解释、肺叶或肺萎陷, 肺野透光度减低, 呈云雾状改变, 或呈弥漫散在的斑点状、片状阴影 ${ }^{[2]}$ 。实验室指标血气分析：2例患者 $\mathrm{Pa} 02 / \mathrm{Fi} 02 \leqslant 100 \mathrm{mmHg}$, 5 例患者 $100 \mathrm{mmHg} \leqslant \mathrm{Pa} 02 / \mathrm{Fi} 02 \leqslant 200 \mathrm{mmHg}$, 9 例患者 $100 \mathrm{mmHg} \leqslant \mathrm{Pa} 02 / \mathrm{Fi} 02 \leqslant 200 \mathrm{mmHg}$, 全部患者均满足 2006 年由中华医学会重 症医学分会所制定的ALI/ARDS标准 ${ }^{[3]}$ 。

\section{3治疗方法}

处理措施：（1）早期补充血容量, 维持患者血压及尿量。合并明显血、 气胸病例则事先行胸腔穿刺或胸腔闭式引流术, 其中行胸腔闭式引流术 6 例。(2) 保持呼吸道通畅, 吸痰, 严密监测生命征, 同时立即行气管插管, 呼 吸机辅助呼吸压力支持模式, 使潮气量控制在 $6-8 \mathrm{~mL} / \mathrm{kg}$, 呼吸频率 $12-18$ 次 $/ \mathrm{min}$, 调节吸呼比在 $1: 2$ 左右: PS : $15-20 \mathrm{mmHg}, \mathrm{PEEP}: 4-8 \mathrm{mmHg}$, 首先吸入氧浓 度为 $60 \%-80 \%$, 根据Pa02值, 从小量开始加用PEEP。在血气分析的各项指标 检测正常, 且Sp02超过 $90 \%$ 时, 逐步降低氧气浓度至30\%-50\%之间。其中每天 早晚两次血气分析检查。患者躁动出现呼吸机抵抗, 给予镇静镇痛治疗, 氧合指数较低患者, 给予彻底打掉自主呼吸, 充分降低患者氧耗。(3) 针对 患者肺部创伤给予抗感染治疗, 在充分的抗感染治疗前提下, 给予激素及 支气管扩张剂, 静脉点滴氨埧索化痰治疗。(4) 使用限制性液体管理策略, 复苏补充液体后, 每日保持出入量平衡, 给予静点白蛋白、血浆, 同时给予 利尿治疗, 利尿后要 1 小时评估一次也出入量, 保证患者利尿过度, 导致患 者低血容量发生。由于患者有显性蒸发及不显性蒸发, 胸腔引流液引出及 痰液引出, 可保证患者入量少于出量, 达到患者限制性液体管理的目标。

\section{2 结果}

16例患者行机械通气支持、限制性液体管理策略及抗炎化痰复苏补液 等治疗, 患者氧合指数每日逐渐好转, 氧饱和度逐渐升高, 呼吸机支持条 件逐渐降低, 即PS、PEEP及氧浓度逐渐降低, 肺部顺应性逐渐好转, 潮气量 增加, 镇静镇痛药物逐渐减量。呼吸困难症状好转。平均带机时间为 $8.5 \mathrm{~d}$, 均顺利脱机拔管转出重症医学病房。

\section{3 讨论}

总结以上病例得出以下体会：（1）在患者创伤后存在大量失血及体液 丧失, 本组患者16例均有不同程度的脱水征象, 表现为尿量减少。体征表现 为全身皮肤水肿, 正是由于创伤后导致的全身炎症反应, 毛细血管渗漏造 成上述结果, 从而导致患者血容量不足, 严重者导致患者低血容量, 循环不 稳定, 重要脏器功能血流灌注不足。乳酸升高, 使患者心肌抑制, 甚至造成 患者心脏骤停。创伤性湿肺导致的肺水肿及肺动脉高压也会影响心脏功能, 严重者出现心力衰竭。所以复苏补液防止心力衰竭很重要, 输液过程中适
当补充血浆及白蛋白, 再给予利尿治疗, 从而减轻肺水肿, 减轻患者心脏负 荷, 预防患者出现心力衰竭。(2) 积极气管插管呼吸机辅助呼吸, 常用的呼 吸末正压通气方式 (PEEP) $5 \sim 10 \mathrm{cmH} 20$, 可有效改善肺泡及间质水肿, 促进 不张肺膨胀, 提高肺顺应性, 保证充分的气体交换。(3) 患者多为重度复合 伤, 肋骨骨折、腹部及骨盆损伤, 导致患者剧烈疼痛, 使患者不能有效咳嗽, 致使气道分泌物蓄积, 出现肺部感染, 此外剧烈疼痛导致患者躁动, 呼吸机 抵抗, 加重呼吸肌做工, 两种因素加重缺氧, 故应积极有效的镇痛镇静, 可 给与瑞芬级咪达唑仑输液洜洜入治疗。(4) 合理应用抗生素预防肺部感染。 肺创伤后一般 3 天后逐渐出现感染, 而早期应用预防是关键, 因患者出现炎 症反应免疫机能较差, 肺部感染一旦出现较难控制。尤其患者已经行气管 插管, 都是严重的感染因素。所以早期要足量使用广谱抗生素, 覆盖球菌杆

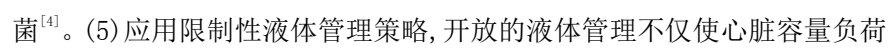
过重, 严重的是导致患者肺部水肿加重, 使创伤性湿肺治疗较困难。我们对 于16例患者均严格控制患者出入量, 每天出入量保持在 $3000 \mathrm{~m} 1$ 左右, 出入 量保持平衡甚至是负平衡 200-500m1, 静点白蛋白及血浆, 同时利尿治疗, 这样消除患者肺水, 保持患者身体处于一种干的状态。综上所述, 创伤性湿 肺是创伤后机体炎症失衡造成的肺部炎症反应, 从而导致的肺间质水肿, 使患者肺部通气功能及弥散功能障碍, 导致一系列的顽固性的低氧血症, 多发生于重大创伤或应激反应后。是一种常见的创伤后并发症。治疗的 关键在于早期发现, 早期确诊, 多学科协助, 积极处理原发伤的同时给予 气管插管及限制性液体管理策略, 创伤后机体组织水肿一般 48-72 小时 水肿高峰, 72 小时至 $7 \mathrm{~d}$ 患者进入水肿回收期。开放的液体管理, 会较容易 造成肺水肿风险, 使原有的呼吸困难级创伤性湿肺病情加重, 当患者处 于水肿回收期, 回心血量增加, 加重患者心脏负担, 使液体管理保持在平 衡或负平衡状态, 应用限制性液体管理策略可防止水肿及水负荷风险。 同时给予合理应用抗生素, 这样才能增加患者脱机拔管率, 缩短患者重 症监护病房治疗时间。

\section{[参考文献]}

[1]Song Chao,Li Haitao,Li Yi,Dai Minhui,Zhang Lemeng,Liu Shuai,Tan Hongyi,Deng Pengbo,Liu Jingjing,Mao Zhi,Li Qian,Su Xiaoli,Long Yuan,Lin Fengyu,Zeng Yanjun,Fan Yifei,Luo Bailing,Hu Chengping,Pan Pinhua. NETS promote ALI/ARDS inflammation by regulating alveolar macrophage polarization.[J]. Experimental ce11 research,2019,382(2).

[2]谭国光.X线、CT检查在创伤性湿肺诊断中的应用 [J].中国继续医学 教育,2019,11(22):84-86.

[3]中华医学会重症医学分会.急性肺损伤/急性呼吸窘迫综合征诊断 和治疗指南[M].中国危重病急救医学,2006,18(12):706-710.

$[4]$ 梁盛辉.收治创伤性湿肺98例临床分析[J].现代医学与健康研究电 子杂志,2018,2(17):162+164. 


\section{二维码技术在术前访视中的应用}

屈卓

辽宁省肿瘤医院

DOI:10.32629/bmtr.v1i1.321

[摘 要] 随着医疗卫生领域科技的发展,移动医疗在临床得到了广泛的应用,包括数据采集,健康管理,疾病监测等。移动医疗(mobile health)是指 使用通信技术如计算机、移动电话和卫星通信等提供医疗和信息 ${ }^{[1]}$ 。近年来,移动医疗也在不断渗入到护理工作中,使护理工作效率得以提高同 时简化患者诊疗程序。本文讨论移动医疗的一种,二维码技术在术前访视中的应用。术前患者通过手机识别二维码可直接在手机查看术前宣教 视频及图文内容,填写术前访视问卷传至系统内,负责该患者护士可直接从系统查看患者信息。这种术前访视形式能够明显的提高医院的术前访 视率,保证术前访视的高质量完成,减轻手术室护士工作负荷,让患者更全面的了解手术相关信息,减轻患者焦虑情绪,降低手术停台率。

[关键词] 术前访视; 护理；二维码

\section{Application of Two-dimensional Code Technology in Preoperative Visits \\ Qu Zhuo}

\section{Liaoning Cancer Hospital \& Institute}

[Abstract] With the development of science and technology in the medical and health field, mobile medicine has been widely used in clinical practice, including data collection, health management, and disease monitoring. Mobile health refers to the use of communication technologies such as computers, mobile phones, and satellite communications to provide medical care and information ${ }^{[1]}$. In recent years, mobile medicine has also been continuously infiltrated into nursing work, which has improved the efficiency of nursing work and simplified the procedures of patient diagnosis and treatment. This article discusses a kind of mobile medical, the application of two-dimensional code technology in preoperative visits. Before the operation, the patient can use the mobile phone to identify the two-dimensional code to view the preoperative education video and graphic content directly on the mobile phone, fill out the preoperative visit questionnaire and transfer it to the system, the nurse in charge of the patient can view the patient information directly from the system. This form of preoperative visits can significantly improve the preoperative visit rate of the hospital, ensure the high-quality completion of preoperative visits, reduce the workload of the nurses in the operating room, allow patients to have a more comprehensive understanding of surgical-related information, and relieve the anxiety of patients and reduce the rate of surgical cessation.

[Keywords] preoperative visit; nursing; two-dimensional code

\section{1 术前访视现状简介}

术前访视是手术室护士的职能和职责之一, 作为手术室护理工作的 一项重要内容, 在我国临床已经开展了十余年 ${ }^{[2]}$ 。现已有许多研究表明术 前访视在缓解患者焦虑, 提高患者手术配合度, 降低手术亭台率, 减少医 患纠纷等方面的显著作用。但是在术前访视的实施过程中仍存在许多困 难。术前患者多, 手术护士人员紧张, 术间内术前访视以强调重点省时高 效为主, 患者未能在病房等候等条件干扰, 致使术前访视率很难达到百 分之百。

\section{2 通过二维码技术辅助进行术前访视}

2. 1 建立术前访视内容及标准

由手术室护士长选取科室内各层级人员组建术前访视小组, 设立组长 专项负责。通过进入临床科室实际调查, 联系病房实际工作共同确定术前 访视内容及调查问卷, 建立术前访视标准流程。术前访视内容应涵盖手术 室的环境介绍, 手术流程, 术前准备及注意事项的介绍, 同时。根具整理内 容制定术前访视宣传手册, 宣传手册内有漫画配图以方便理解。内容确定 后制定二维码, 二维码应达到识别后可见手术室术前访视宣教视频, 术前 访视图文内容及调查问卷。将二维码分发至各外科病房, 放置于护士站, 术前患者可及时识别二维码观看并填写问卷, 以方便手术室护士了解患者 术前需知晓的自然情况。

2. 2 识别二维码术前访视模式推广
首先由手术室护士长联系外科各病房护士长获得病房配合, 术前患者 由责护告知识别二维码即可了解术前注意事项等内容。同时院内公众号发 文推广, 让更多的人知道有这种术前访视方式存在, 为解决患者及家属对 手术的疑问提供便利方法。术前访视小组成员到临床定期到临床推广随访, 向临床患者讲解如何用手机扫二维码进行术前访视, 并解读术前访视的重 要性。同时统计临床术前患者对二维码识别式术前访视的使用在操作方 法、使用效果、患者观看后的理解程度, 随时接收意见建议, 发现问题进行 整改。并且与原有的护士进入病房进行术前访视的方式进行对比调查, 统 一整合后进行修改。

2. 3护士面对面术前访视共同作业

通过样本观察, 医护人员以术前访视的方式对病人进行有效激励, 可 以显著提高病人的信心, 并且使得病人积极配合医院的治疗周期, 从而使 得病人康复率得 到明显提升。并且病情较重的患者反馈术前与手术室护 士和麻醉医师进行面对面的交谈使他们更加觉得有安全感。因而对于手术 复杂, 患者身体状态或者心理状态差的患者, 仍继续实施面对面模式术前 访视。通过直观的评估, 为患者制定更好的护理方案, 在术前了解充分以保 证手术顺利进行 ${ }^{[3]}$ 。二维码术前访视技术并不能完全取替护士进行术前访 视, 但是此项技术大大的改善了术前访视的状态, 为患者提供了更加方便 的了解方式, 同时也是跟上新时代脚步所必须的产物。护士面对面术前访 视与二维码术前访视共同作业, 才能使术前访视工作更加完善。 